\title{
Contrast-enhanced ultrasound aids in the detection of prostate rhabdomyosarcoma: A case report and literature review
}

\author{
LE-HANG GUO, HUI-XIONG XU, HUI-JUN FU, LI-PING SUN and BO-JI LIU \\ Department of Medical Ultrasound, Shanghai Tenth People's Hospital of Tongji University, Shanghai 200072, P.R. China
}

Received September 3, 2014; Accepted May 20, 2015

DOI: $10.3892 / \mathrm{ol} .2015 .3449$

\begin{abstract}
Prostate sarcoma is a rare malignancy with an extremely poor prognosis. The extremely low morbidity and atypical clinical symptoms contribute to a missed diagnosis. The typical features of prostate sarcoma in transrectal ultrasound (US) and magnetic resonance imaging, such as a markedly enlarged volume and irregular prostatic contours, cannot usually be found until dysuria or even uroschesis occurs, and may then be too late to treat. However, there appears to no specific tumor marker for the disease in the serum. The present study reports a case of a young male patient who was diagnosed with prostate rhabdomyosarcoma. This was, to the best of our knowledge, the first case of this diagnosis using contrast-enhanced US (CEUS) when the symptoms were not severe. In this case, the intralesional non-enhancement areas and rim-like hyper-enhancement around the lesion were considered to be the main CEUS features of prostate rhabdomyosarcoma. The present study also reviews the associated literature.
\end{abstract}

\section{Introduction}

Prostate sarcoma is a rare malignancy accounting for $<1 \%$ of all primary prostate malignancies in adults (1). It has an extremely poor prognosis, with a median overall survival time of 23 months, partly due to the difficulty of early detection. The early clinical symptoms, such as dysuria or abdomen pain, are unspecific and there is no specific serum marker for the entity, thus it is always firstly detected by imaging (2). Imaging-guided biopsy is the standard diagnostic technique used to identify prostate sarcoma (3). In recent years, contrast-enhanced ultrasound (CEUS), which can depict the micro- and macro-vascularity of prostate, has been proved effective in detecting prostate adenocarcinoma $(4,5)$. However,

Correspondence to: Professor Hui-Xiong $\mathrm{Xu}$, Department of Medical Ultrasound, Shanghai Tenth People's Hospital of Tongji University, 301 Yanchangzhong Road, Shanghai 200072, P.R. China E-mail: xuhuixiong@hotmail.com

Key words: prostate rhabdomyosarcoma, contrast-enhanced ultrasound, ultrasound to the best of our knowledge, the CEUS features of prostate sarcoma remain unknown. Thus, the current study presents a case of prostate rhabdomyosarcoma, with emphasis on the CEUS findings. The associated literature on prostate sarcoma is also reviewed. Written informed consent was obtained from the patient.

\section{Case report}

A 33-year-old male was referred to the Department of Ultrasound (Shanghai Tenth People's Hospital, Shanghai, China) in March 2014 due to frequent micturition, accompanied with a low-grade fever $\left(37.5^{\circ} \mathrm{C}\right)$ and lower abdomen pain. Prior to this, the patient had been treated for prostatitis in a community hospital for 3 months, but without evident remission. No abnormal laboratory test findings were recorded, with the exception of a grade of 1+ for urinary occult blood upon urinalysis. The prostate-specific antigen (PSA) level $(1.26 \mathrm{ng} / \mathrm{ml})$ was within normal limits. Other symptoms, such as frequent micturition and a poor urinary stream were present occasionally. Local stenosis of the rectum was suspected upon digital rectal examination. There was no family history of genitourinary cancer.

Transrectal US (TRUS) was performed with a LOGIQ E9 scanner (GE Healthcare, Milwaukee, WI, USA), which was equipped with a transrectal transducer (E8C; 5-9 MHz). The patient was examined in the left recumbent position, with slightly bent knees. The prostate was enlarged asymmetrically on gray-scale US imaging, measuring $\sim 7.0 \times 5.1 \times 5.2 \mathrm{~cm}$ in size. The volume (V) of the prostate was computed to be $97 \mathrm{ml}$ when using the following formula: $\mathrm{V}=\pi \times \mathrm{L} \times \mathrm{W} \times \mathrm{H} / 6$, where $\mathrm{L}$ is the length, $\mathrm{W}$ is the width and $\mathrm{H}$ is the height of the prostate. The left lobe of the prostate was protruding with a well-delineated margin, and the left lobe was markedly larger (volume, $78 \mathrm{ml}$ ) than the right lobe (volume, $18 \mathrm{ml}$ ). The prostatic urethra and ejaculatory ducts were pushed to the right and were not clearly shown (Fig. 1A). The left lobe was heterogeneous in echogenicity on US, with irregular small hypoechoic areas. Color Doppler imaging showed dotted blood flow within the left lobe (Fig. 1B).

Transrectal elastography was performed with the same scanner to provide information on stiffness. The peripheral zone of the left lobe was displayed in blue, indicating tissue components with relatively hard stiffness. The central zone of the left lobe was mainly displayed green (i.e., intermediate stiffness), 

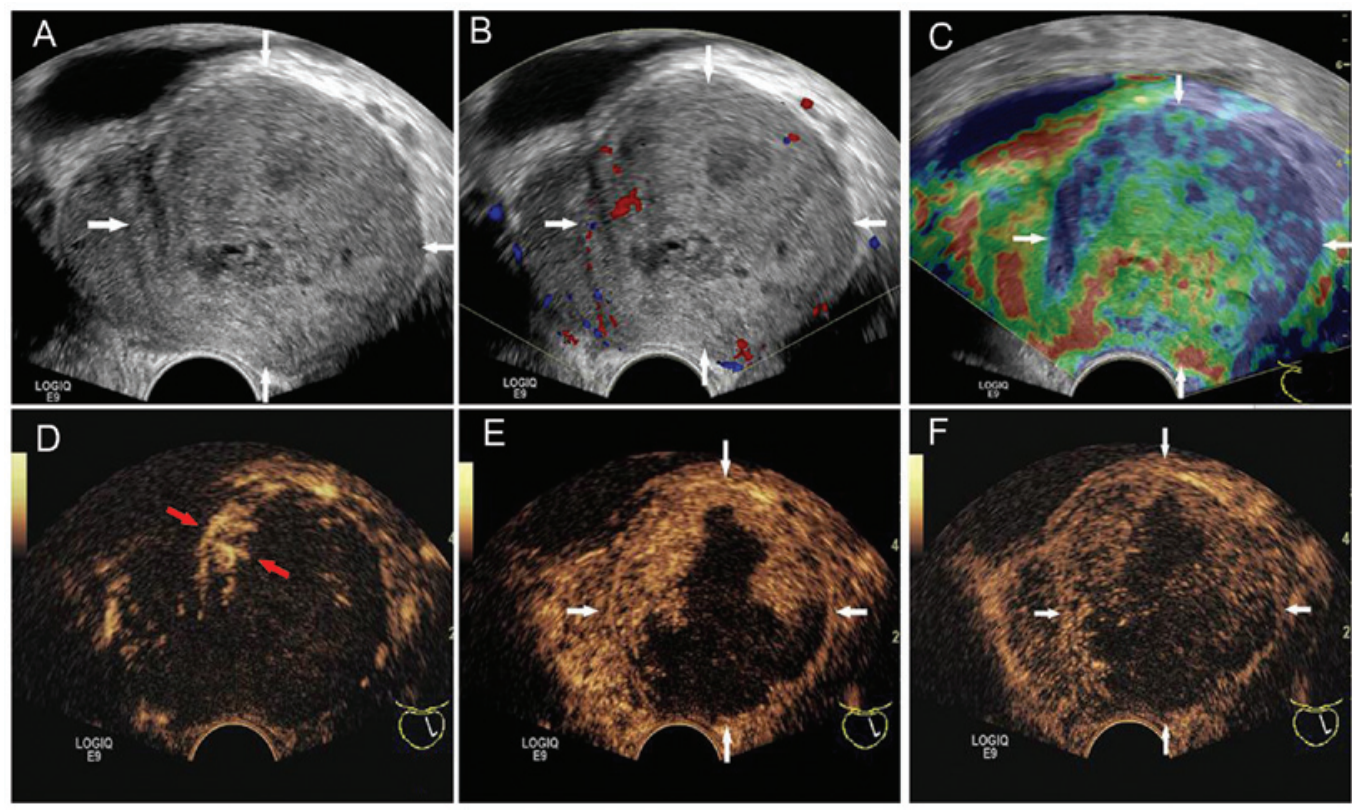

Figure 1. Transverse directional views of the prostatic left lobe on transrectal US (white arrow). (A) Gray-scale US: An enlarged prostate with irregular internal hypoechoic regions. The distorted middle line was also shown, but the prostate urethra and ejaculatory ducts were not clear. (B) Color doppler imaging: Dotted blood flow within the left lobe. (C) Elastography: The peripheral zone of the left lobe was displayed in blue, and the central zone of the left lobe was mainly displayed in green, with red patch-like areas. (D) Arterial phase $(11 \mathrm{sec})$ : Enhancement started from the edge of the left lobe (red arrow). (E) Venous phase $(52 \mathrm{sec})$ : Contrast agent had extended inward the left lobe and formed hyper-enhancement zones, but had not extended into the other region. (F) Late phase $(150 \mathrm{sec})$ : Contrast agent washed out slowly and had not extended inward the non-enhancement zone. US, ultrasound.
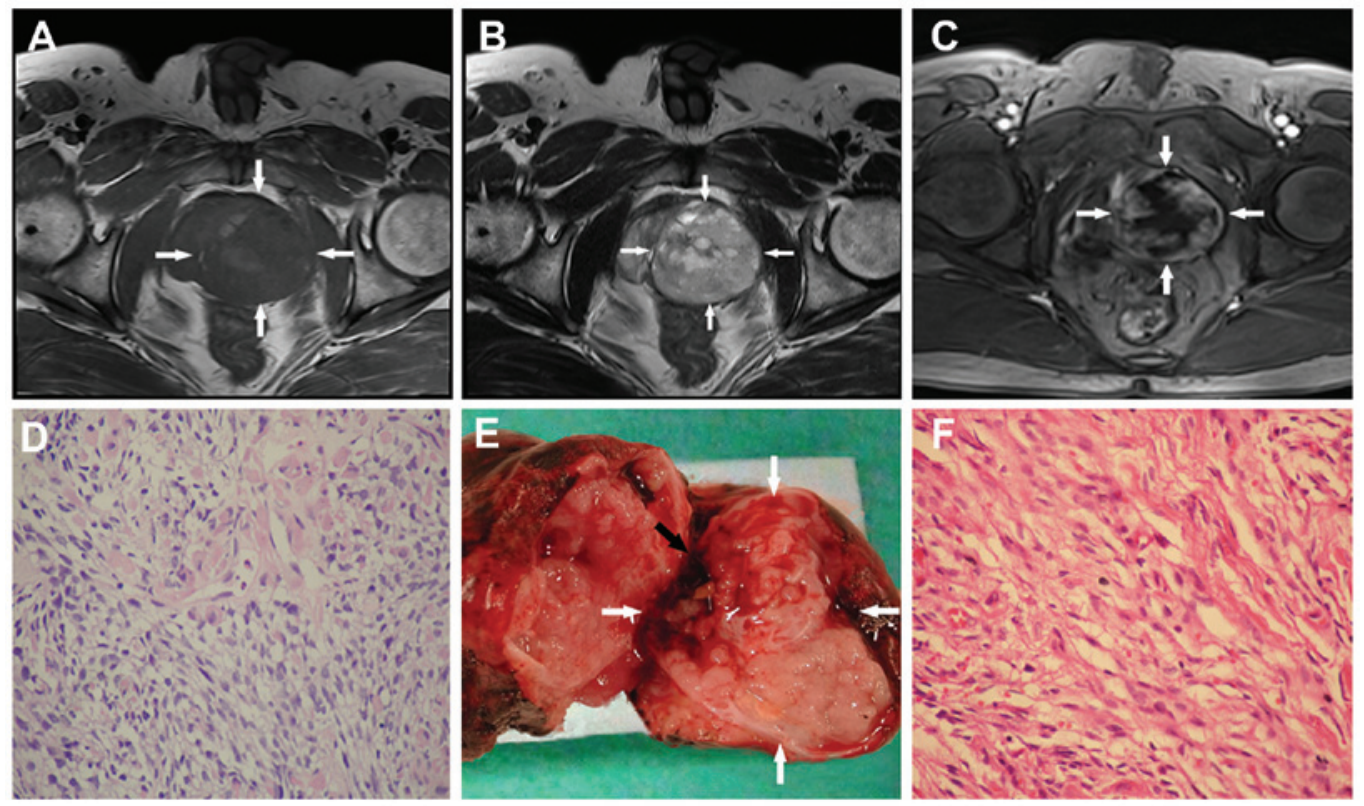

Figure 2. Transverse directional views of a mass on magnetic resonance imaging (MRI) and the pathological gross specimens (white arrow). (A) T1-weighted image: A well-defined and large mass was detected in the pelvis, with isointense signals (white arrow). (B) T2-weighted image: A well-defined and large mass was detected in the pelvis, with increased signals and more marked heterogeneity (white arrow). (C) Contrast-enhanced MRI: The heterogeneous hyper-enhancement was similar with contrast-enhanced ultrasound (CEUS; white arrow). (D) Light microscopic examination for biopsy: Fascicles of primitive, undifferentiated and diffusely distributed spindle cells [hematoxylin and eosin (HE) staining; magnification, x40]. (E) Pathological gross specimens: The tumor specimen (cut open) was soft, fragile and moist, with local internal hemorrhage (black arrow), exactly where CEUS contrast agent started to diffuse into the tumor. A pseudo-capsule wrapped around the tumor was also observed (white arrow). (F) Light microscopic examination for gross specimens: Hypercellular mesenchymal tumor composed of strap and tadpole cells with eosinophilic fibrillary cytoplasm (HE staining; magnification, x100).

with red or yellow patch-like areas (i.e., soft or low stiffness). The right lobe was mainly displayed in green (Fig. 1C).

CEUS was performed subsequent to the injection of $2.4 \mathrm{ml}$ contrast agent (SonoVue, Bracco, Milan, Italy) followed by
$10 \mathrm{ml}$ of normal saline flush through the antecubital vein. Early arterial enhancement started from the edge of the left lobe at $11 \mathrm{sec}$ after contrast injection. There was rim-like hyper-enhancement surrounding the left lobe (Fig. 1D). 
Following this, the contrast agent extended immediately inward and formed a number of hyper-enhancement zones that were irregular, but not isolated (Fig. 1E). The contrast agent washed out slowly and the hyper-enhancement lasted during the whole arterial $(<30 \mathrm{sec})$, venous $(31-120 \mathrm{sec})$ and late $(121-180 \mathrm{sec})$ phases. Adversely, contrast agent never extended into the other region of the left lobe (Fig. 1F). The non-enhancement area was mainly at the center and posterior of the left lobe, with an irregular shape, which corresponded to the intermediate and low elasticity areas on elastography examination, and the irregular hypoechoic areas on baseline US.

Based on the results of CEUS, the 'left lobe' was supposed to be a rare malignant neoplasm with high confidence. Hence, the patient was admitted to hospital immediately and a biopsy was performed. Only 4 samples were obtained ( 2 from the upper pole and 2 from the lower pole), which were pale and fragile, with high moisture contents.

Prior to the biopsy, a series of pre-operative examinations, including abdominal and pelvic magnetic resonance imaging (MRI), chest radiographs, bone scintigraphy and certain serum tests, had been performed to evaluate the tumor staging. The MRI depicted a pelvic mass measuring $\sim 5.4 \times 5.6 \times 5 \mathrm{~cm}$ in size, which was well defined, pushed the urethra to the right and compressed the left seminal vesicle observably. The tumor revealed isointense signals on $\mathrm{T}_{1}$-weighted imaging (Fig. 2A) and increased signal intensity on $\mathrm{T}_{2}$-weighted imaging, with heterogeneity (Fig. 2B). Multiple small high signal intensity areas distributed inside the mass were considered to be cysts. Following intravenous gadolinium administration, heterogeneous hyper-enhancement was noted (Fig. 2C). The diagnosis was a malignant lesion in the pelvic cavity, without a precise location, and no abnormal iliac lymph nodes were noted. The remaining scans and serum tests negative.

The biopsy samples were analyzed by an experienced pathologist and showed mixed bundles of primitive, undifferentiated and diffusely distributed cells. Cytological atypia was moderate, but the mitotic activity was high. Necrotic tissues were noted in these samples (Fig. 2D). Immunohistochemical studies showed that the neoplastic cells were positive for muscle-specific actin, fast myosin and vimentin, whereas the cells were negative for desmin, keratin AE1/AE3, cytokeratin 5/6, PSA and prostate-specific acid phosphatase. All pathological manifestations proved that the patient had a subtype of prostate sarcoma.

The patient underwent a radical prostatectomy. The whole prostate, seminal vesicles, urethral bulb and soft tissue adjacent to the prostate were totally removed. Macroscopically, the complete tumor was soft, fragile, moist with internal hemorrhage and encased by a pseudo-capsule (Fig. 2E). The final pathological diagnosis was of a rhabdomyosarcoma (Fig. 2F) with large necrotic areas. At 12 days post-surgery, the patient recovered well and was discharged in a stable condition. To date, the patient has finished 8 courses of chemotherapy (cisplatin and bleomycin were administered using bladder irrigation) and is alive with no signs of recurrence or metastases.

\section{Discussion}

Adult prostate sarcoma is extremely rare, with a morbidity rate of $0.1-0.2 \%$ in all prostate tumors (6), which is far below the morbidity rate of prostate adenocarcinoma (7). The main subtypes of prostate sarcoma consist of leiomyosarcoma, rhabdomyosarcoma, synovial sarcoma, fibrosarcoma, spindle cell sarcoma, prostatic stromal sarcoma and undifferentiated sarcoma (6). Rhabdomyosarcoma usually occurs in young men, particularly in children as the most common subtype (8). This high-grade malignant tumor with a dismal prognosis grows fast and invasively (6), and is prone to bleeding, necrosis or cystic degeneration. Sarcomas often metastasize hematogenously in the early stage due to the nutrient-rich blood supply (9). Early diagnosis and surgical resection with a curative intent offers patients the best chance of survival (10).

However, for the majority of urologists or radiologists, their experience of this diagnosis is limited. The initial clinical symptoms are usually unspecific unless urinary obstruction may increase the clinician's vigilance (11). Additionally, there appears to be no specific tumor marker for sarcoma in the serum (12). Due to these issues, an early diagnosis is extremely difficult, resulting in a dismal prognosis (2).

Ultrasound, MRI and cytology findings in the urine are reported as methods for the detection of prostate sarcoma, On TRUS, a markedly enlarged volume and irregular margins are important characteristics of prostate sarcoma $(13,14)$. In addition, TRUS is usually used to guide a biopsy to confirm the diagnosis (1). The imaging features of MRI, such as a large size, irregular margins and heterogeneity, are similar to those of TRUS $(15,16)$. Besides that, it has an advantage in detecting metastatic lesions and revealing the adjacent structures (17). Cytology findings in the urine may be useful, but are also varied, therefore their use is not indicated as a routine procedure (18).

In the present case, the volume and margin of the tumor on TRUS were not typical, as previously reported $(3,13)$. Due to the benefits of using CEUS, unusual features such as intralesional non-enhancement areas and rim-like hyper-enhancement around the lesion were revealed, so that timely and effective treatment was performed. In addition, only 4 biopsy samples were obtained from the hyper-enhancement areas on CEUS. This avoided the unnecessary trauma to the patient and the risk of metastasis whenever possible. In the majority of situations, at least 12 cores will be sampled due to the large volume (19).

As aforementioned, due to the extremely low morbidity of prostate sarcoma, it is not necessary to perform CEUS routinely. Under certain situations, for example, a markedly enlarged volume, an irregular and asymmetrical appearance, marked heterogeneity internally or other abnormal signs in the prostate, CEUS may be useful as a rapid diagnostic test, particularly in young patients. Likewise, CEUS is useful to determine the biopsy sites to decrease any unnecessary trauma and risks of metastasis. Surgery, chemotherapy and radiotherapy are the main methods of treatment. Among them, surgical resection is the most recommended. In conclusion, this present case of prostate rhabdomyosarcoma benefitted from the application of CEUS.

\section{Acknowledgements}

This study was supported in part by grants from the Key Project of the Shanghai Health Bureau (no. 20114003) and the Shanghai Talent Development Project of the Shanghai Human Resource and Social Security Bureau (no. 2012045). 


\section{References}

1. Janet NL, May AW and Akins RS: Sarcoma of the prostate: A single institutional review. Am J Clin Oncol 32: 27-29, 2009.

2. Sexton WJ, Lance RE, Reyes AO, Pisters PW, Tu SM and Pisters LL: Adult prostate sarcoma: The M. D. Anderson Cancer Center Experience. J Urol 166: 521-525, 2001.

3. Wang X, Liu L, Tang H, Rao Z, Zhan W, Li X, Zeng H, Zhang P, Wei B, Lin T, et al: Twenty-five cases of adult prostate sarcoma treated at a high-volume institution from 1989 to 2009. Urology 82: 160-165, 2013.

4. Xie SW, Li HL, Du J, Xia JG, Guo YF, Xin M and Li FH: Contrast-enhanced ultrasonography with contrast-tuned imaging technology for the detection of prostate cancer: Comparison with conventional ultrasonography. BJU Int 109: 1620-1626, 2012.

5. Zhao HW, Luo JH, Xu HX, Wang DH, Lai YR, Chen MN, Lv JY, Xie XY, Lu MD and Chen W: The value of contrast-enhanced transrectal ultrasound in predicting the nature of prostate diseases and the Gleason score of prostate cancer by a subjective blood flow grading scale. Urol Int 87: 165-170, 2011.

6. Kim JY, Cho YM and Ro JY: Prostatic stromal sarcoma with rhabdoid features. Ann Diagn Pathol 14: 453-456, 2010.

7. Siegel R, Naishadham D and Jemal A: Cancer statistics, 2013. CA Cancer J Clin 63: 11-30, 2013.

8. Perez EA, Kassira N, Cheung MC, Koniaris LG, Neville HL and Sola JE: Rhabdomyosarcoma in children: A SEER population based study. J Surg Res 170: e243-e251, 2011.

9. Dundore PA, Cheville JC, Nascimento AG, Farrow GM and Bostwick DG: Carcinosarcoma of the prostate. Report of 21 cases. Cancer 76: 1035-1042, 1995.

10. Bisceglia M, Magro G, Carosi I, Cannazza V and Ben Dor D: Primary embryonal rhabdomyosarcoma of the prostate in adults: Report of a case and review of the literature. Int J Surg Pathol 19: 831-837, 2011.
11. Mukouyama H, Sugaya K, Ogawa Y, Koyama Y, Hatano T and Toda T: Poorly differentiated sarcoma of the prostate causing obstructive acute renal failure: A case report. Int J Urol 6: 615-619, 1999.

12. Rogers CG, Parwani A, Tekes A, Schoenberg MP and Epstein JI: Carcinosarcoma of the prostate with urothelial and squamous components. J Urol 173: 439-440, 2005.

13. Stilgenbauer R, Benedict M, Bamshad R and Viduetsky A: Sarcoma of the prostate: Sonographic findings and pathologic correlation. J Ultrasound Med 26: 1789-1793, 2007.

14. Terris MK: Transrectal ultrasound appearance of radiation-induced prostatic sarcoma. Prostate 37: 182-186, 1998.

15. Cheng YC, Wang JH, Shen SH, Chang YH, Chen PC, Pan CC and Chang CY: MRI findings of prostatic synovial sarcoma. Br J Radiol 80: e15-e18, 2007.

16. Ren FY, Lu JP, Wang J, Ye JJ, Shao CW and Wang MJ: Adult prostate sarcoma: Radiological-clinical correlation. Clin Radiol 64: 171-177, 2009.

17. ESMO/European Sarcoma Network Working Group: ESMO Clinical Practice Guidelines for diagnosis, treatment and follow-up. Ann Oncol 23 (Suppl 7): vii92-vii99, 2012.

18. Stoll LM, Johnson MW and Rosenthal DL: High-grade prostatic sarcoma seen in a catheterized urine specimen: Case report and differential diagnosis. Diagn Cytopathol 39: 762-766, 2011.

19. Heidenreich A, Bastian PJ, Bellmunt J, Bolla M, Joniau S, van der Kwast T, Mason M, Matveev V, Wiegel T, Zattoni F, et al: European Association of Urology: EAU guidelines on prostate cancer. Part 1: Screening, diagnosis and local treatment with Curative Intent-Update 2013. Eur Urol 65: 124-137, 2014. 